\title{
Identification and structural features analysis of long noncoding RNAs
}

\author{
Pronozin A.*, Afonnikov D. \\ Kurchatov Genomic Center of the Institute of Cytology and Genetics of Siberian Branch of the Russian \\ Academy of Sciences, Novosibirsk, Russia \\ * email: pronozinartem95@gmail.com
}

Motivation and Aim: Long non-coding RNAs (lncRNAs) are typically defined as transcripts of more than 200 nucleotides length and without any protein coding potential. The functions are poorly understood, however, a number of well-known plant lncRNAs play diverse roles in $\mathrm{X}$ inactivation, imprinting and gene expression. Thus lncRNAs are involved in important plant development processes such as phosphate homeostasis, flowering, photomorphogenesis and stress response in this connection, their study is relevant. Information is obtained from transcriptomes, but bioinformatic annotation methods are not sufficiently presented, especially for plants. This raises the challenge of developing approaches to automatic annotation and prediction of lncRNA functions in plants.

Methods and Algorithms: In this paper a computational pipeline for the identification and annotation of lncRNA in the plant transcriptome has been developed, steps:

1. Identification of lncRNAs - lncFinder [1].

2. Alignment lncRNA on referense genome - GMAP [2].

3. IncRNAs classification - gffcompare [3]. IncRNAs structural features analysis. The pipeline is implemented using the Snakemake workflow management system language. Results: The pipeline was used to analyze $~ 800$ Zea mays transcriptomes comprising 3148430 transcripts in total. We identified 2741504 (87\%) lncRNAs; of them $98 \%$ were aligned to the reference genome. We identified 334069 exon antisense, 4390 intron antisense, 231970 multi-exon, 81163 retained introns, 512753 intergenic. Antisense lncRNAs alignment (exon, intron, 338459 tr.) on structure of the target gene, showed that the predominant amount of lncRNA is aligned on exon 1 of the target gene.

Conclusion: The proposed pipeline made it possible to identify 1164345 new lncRNAs in the maize genome, annotate them and evaluate their structural features.

Acknowledgements: Work was funded by the Kurchatov Genomic Center of the Institute of Cytology and Genetics, SB RAS, agreement with the Ministry of Education and Science of the Russian Federation No. 075-15-2019-1662.

\section{References}

1 Han S. et al. LncFinder: an integrated platform for long non-coding RNA identification utilizing sequence intrinsic composition, structural information and physicochemical property. Briefings in Bioinformatics. 2019;20(6):2009-2027.

2 Wu T. D., Watanabe C. K. GMAP: a genomic mapping and alignment program for mRNA and EST sequences. Bioinformatics. 2005;21(9):1859-1875.

3 Pertea G., Pertea M. GFF utilities: GffRead and GffCompare. F1000Research. 2020;9. 